\title{
Soliton dynamics in integrated photonic chips
}

\author{
Chad Husko $^{1}$, Matthias Wulf ${ }^{2}$, L. Kobus Kuipers ${ }^{2}$, Thomas F. Krauss ${ }^{3}$, Chee Wei \\ Wong $^{4}$, Pierre Colman ${ }^{5}$, Sylvain Combrié ${ }^{6}$, Alfredo De Rossi ${ }^{6}$, Andrea \\ Blanco-Redondo ${ }^{7}$, Simon Lefrancois ${ }^{7}$, Benjamin J. Eggleton ${ }^{7}$ \\ ${ }^{1}$ Center for Nanoscale Materials, Argonne National Laboratory, Argonne, Illinois 60439, USA \\ ${ }^{2}$ Center for Nanophotonics, FOM Institute AMOLF, Science Park 104, 1098 XG, Amsterdam, The Netherlands \\ ${ }^{3}$ Department of Physics, University of York, York YO10 5DD, UK \\ ${ }^{4}$ Mesoscopic Optics \& Quantum Electronics Laboratory, University of California, Los Angeles, 90095, USA \\ ${ }^{5}$ Institut d'Electronique Fontamentale (IEF), Universite Paris-Sud, Orsay, France \\ ${ }^{6}$ Thales Research and Technology, 1 Av. A. Fresnel 128, 91767 Palaiseau, France \\ ${ }^{7}$ Centre for Ultrahigh bandwidth Devices for Optical Systems (CUDOS), Institute of Photonics and Optical Science \\ (IPOS), School of Physics, The University of Sydney, NSW 2006, Australia \\ E-mail: chusko@anl.gov
}

\begin{abstract}
In this talk I focus on nonlinear pulses in nanophotonic semiconductor waveguides. Our time-resolved measurements reveal physical phenomena unique to solitons in a free-carrier medium. We support these results with analytic and numerical models.
\end{abstract}

OCIS codes: 130.4310 nonlinear integrated optics;190.4390 Nonlinear optics, integrated optics

\section{Introduction}

Solitons are localized waves formed by a balance of focusing and defocusing effects. These nonlinear waves exist in diverse forms of matter yet exhibit similar properties including stability, periodic recurrence, and particle-like evolution. In the optical domain a temporal soliton can form from the controlled balance of the nonlinear Kerr effect with linear group-velocity dispersion (GVD) [1]. In semiconductor materials one must also account for nonlinear absorption and free carriers at typical intensity levels [2]. The nonlinear absorption attenuates the peak power, restricting the desirable nonlinear phase change due to the Kerr effect. In addition, the nonlinear absorption generates free carriers. The free-carrier plasma modifies the nonlinear pulse evolution with both dispersive (FCD, $n_{F C}$ ) and absorptive (FCA, $\sigma)$ contributions leading to non-trivial dynamics unavailable in other optical systems. For example, while in the spectral domain optical pulses undergo a spectral blueshift due to FCD [3], in contrast the temporal properties are governed by the dynamic interaction of FCD and dispersion together leading to, for example, nonlinear pulse temporal broadening [4]. These free-carrier effects also interplay with and modulate the classical soliton evolution. Temporal solitons in semiconductors have been shown [5,6] including soliton self-frequency blueshift [7] and soliton acceleration [8,9]. In this talk we describe the dynamics unique to solitons in semiconductors through experiment, theory, and modeling.

\section{Solitons in semiconductor photonic crystal waveguides}

Bright solitons are one class of analytic solutions to the nonlinear Schrödinger equation (NLSE) with Kerr nonlinearity $(\gamma)$ and anomalous GVD $\left(\beta_{2}<0\right)$. Fig. 1(a) summarizes the key parameters in the various platforms in which temporal solitons have been demonstrated. The red circle indicates the region of photonic crystal waveguides that we use in these experiments. In photonic crystal waveguides the Kerr nonlinearity is enhanced by the square of the group index [10]. Fig. 1(b) summarizes experimental autocorrelation results demonstrating the temporal evolution of optical solitons in a semiconductor photonic chip. As the pulse energy increases, the output pulse returns to the input duration before further compressing to a minimum duration of 800 fs (deconvolution factor of 1.54 for sech), limited by the laser output power. These on-chip results follow traditional soliton theory with good fidelity. Recently, we demonstrated a new class of 'pure-quartic solitons' with remarkably different properties arising from fourth-order dispersion and the Kerr effect [16]. We next examine solitons with perturbations.

\subsection{Solitons with perturbations}

The illustrations in Fig. 2 provide an insight into soliton perturbations in both glass and semiconductor media. The left side of the figure describes intrapulse Raman scattering which plays an important role in glass media [11]. These effects are known and we refer the reader to the literature for more detail. The right side of the figure describe intrapulse 
(a)

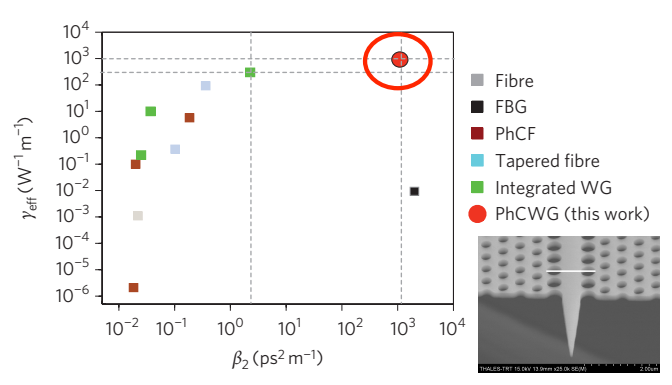

(b) GalnP

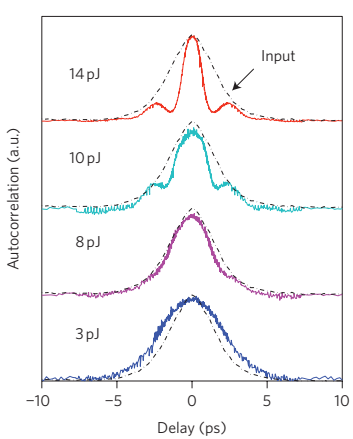

Fig. 1. Temporal optical solitons in photonic crystal waveguides. (a) Nonlinear $\left(\gamma_{e f f}\right)$ and GVD $\left(\beta_{2}\right)$ parameters of various soliton experiments. (b) Autocorrelation measurements demonstrating characteristic higher-soliton behavior. We use GaInP, which suppresses two-photon absorption.
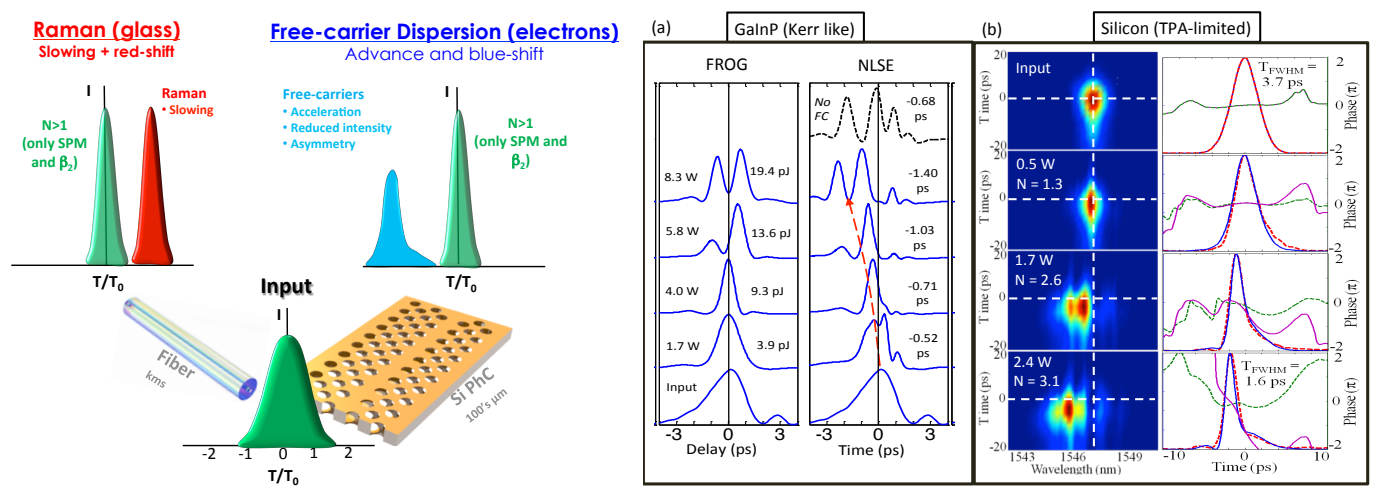

Fig. 2. Solitons with perturbations.Left. Schematic illustrating the differences between soliton evolution in glass (Raman) and semiconductors (free carriers). Right. (a) Weak free carrier regime in GaInP (Kerr + 3PA). (b) Strong free carrier regime in silicon (Kerr + TPA). Details are in the text.

free-carrier effects typical of the temporal behavior in semiconductor waveguides. Owing to free-carrier frequency blue shift from the FCD, the pulse undergoes acceleration in the anomalous medium and arrives before the canonical soliton. The strength of these effects depends on the pulse duration. Whereas Raman processes typically require femtosecond pulses, in contrast, free-carrier effects require longer pulses in time. Physically, this is explained by considering that the energy in the pulse tail needs to experience the free carriers generated by the leading edge. Mathematically, the rate equation describing free-carrier generation involves an integral corresponding to the accumulation of free carriers across the pulse [12]. We now show experiments demonstrating temporal solitons in which free-carriers play a significant role.

\subsection{Weak free carrier regime (3PA-limited)}

While Fig. 1 shows a good first experiment, autocorrelations are symmetric and require an approximation of the pulse shape. As not all pulse dynamics are symmetric, information can be obscured and it is desirable to obtain the real electric-field envelope. With this motivation, we constructed a frequency-resolved optical gating (FROG) apparatus to measure the pulse intensity and phase in both the temporal and the spectral domains. Figure 2(a) shows the retrieved temporal intensity (solid blue) and phase (dashed magenta) of the experimental spectrograms as a function of pulse power. We observe temporal narrowing characteristic of higher-order solitons, as well as the temporal separation 
indicating the onset of periodic evolution. To understand the experiment better we conducted NLSE modeling of the experiment shown in the right panel. As we increase the pulse energy the pulse shifts forward from $0 \mathrm{ps}$ to $-1.4 \mathrm{ps}$, indicating that the pulse advances in time. The black dashed line indicates simulations with suppressed FCD showing the pulse shifts noticeably less in this case and we conclude that FCD causes the temporal advance. We suspect the remaining shift might be due to strong self-steepening in the photonic crystal [13] or residual pulse chirp. More recently we showed that FCD can induce soliton fission [14]. These results of solitons in the weak free-carrier regime encouraged us to study this effect in a TPA-limited material where free carriers play a more significant role.

\subsection{Strong free carrier regime (TPA-limited)}

For this case, we constructed an electrically gated cross-correlation (X-FREG) [15]. Figure 2(b)(left) shows spectrograms at three different coupled power levels for silicon waveguides [9]. The spectrograms clearly reveal the pulse spectrally blue-shifting with increasing power due to larger free-carrier concentrations. Figure 2(b)(right) shows the experimentally retrieved pulse intensities in the time domain indicated as the dashed red lines with NLSE modeling (blue line). We observe compression of 3.7-ps pulses to $1.6 \mathrm{ps}$ (compression factor $\chi_{c}=2.3$ ). It is not obvious how much additional compression is possible in this system due to the presence of TPA. Further, we directly measure the time advance of pulses in the time-of-flight configuration.

\section{References}

1. L. F. Mollenauer, R. H. Stolen, and J. P. Gordon, "Experimental observation of picosecond pulse narrowing and solitons in optical fibers," Phys. Rev. Lett. 45, 1095-1098 (1980).

2. L. Yin and G. P. Agrawal, "Impact of two-photon absorption on self-phase modulation in silicon waveguides." Opt. Lett. 32, 2031-3 (2007).

3. W. Wood, C. Siders, and M. Downer, "Measurement of femtosecond ionization dynamics of atmospheric density gases by spectral blueshifting." Phys. Rev. Lett. 67, 3523-3526 (1991).

4. A. Blanco-Redondo, D. Eades, J. Li, T. F. Krauss, B. J. Eggleton, and C. Husko, "Controlling free-carrier temporal effects in silicon by dispersion engineering," Optica 1, 299-306 (2014).

5. P. Colman, C. Husko, S. Combrié, I. Sagnes, C. W. Wong, and A. De Rossi, "Temporal solitons and pulse compression in photonic crystal waveguides," Nat. Photon. 4, 862-868 (2010).

6. W. Ding, A. V. Gorbach, W. J. Wadswarth, J. C. Knight, D. V. Skryabin, M. J. Strain, M. Sorel, and R. M. De La Rue, "Time and frequency domain measurements of solitons in subwavelength silicon waveguides using a cross-correlation technique." Optics Express 18, 26625-30 (2010).

7. M. Saleh, W. Chang, P. Hölzer, A. Nazarkin, J. Travers, N. Joly, P. Russell, and F. Biancalana, "Theory of Photoionization-Induced Blueshift of Ultrashort Solitons in Gas-Filled Hollow-Core Photonic Crystal Fibers," Phys. Rev. Lett. 107, 203902 (2011).

8. C. Husko, S. Combrié, P. Colman, J. Zheng, A. De Rossi, and C. W. Wong, "Soliton dynamics in the multiphoton plasma regime." Sci. Rep. 3, 1100 (2013).

9. A. Blanco-Redondo, C. Husko, D. Eades, Y. Zhang, J. Li, T. F. Krauss, and B. J. Eggleton, "Observation of soliton compression in silicon photonic crystals," Nat. Commun. 5, 3160 (2014).

10. N. A. R. Bhat and J. E. Sipe, "Optical pulse propagation in nonlinear photonic crystals," Phys. Rev. E 64, 056604 (2001).

11. J. P. Gordon, "Theory of the soliton self-frequency shift." Opt. Lett. 11, 662-664 (1986).

12. S. Lefrancois, C. Husko, A. Blanco-Redondo, and B. J. Eggleton, "Nonlinear silicon photonics analyzed with the moment method," J. Opt. Soc. Am. B 32, 218 (2015).

13. C. Husko and P. Colman, "Giant anomalous self-steepening in photonic crystal waveguides," Phys. Rev. A 92, 013816 (2015).

14. C. Husko, M. Wulf, S. Lefrancois, S. Combrié, G. Lehoucq, A. De Rossi, B. J. Eggleton, and L. Kuipers, "Freecarrier-induced soliton fission unveiled by in situ measurements in nanophotonic waveguides," Nat. Commun. 7, 11332 (2016).

15. B. Thomsen, M. Roelens, R. Watts, and D. Richardson, "Comparison between nonlinear and linear spectrographic techniques for the complete characterization of high bit-rate pulses used in optical communications," IEEE Phot. Tech. Lett. 17, 1914-1916 (2005).

16. A. Blanco-Redondo, C. M. de Sterke, J. E. Sipe, T. F. Krauss, B. J. Eggleton, and C. Husko, "Pure-quartic solitons," Nat. Commun. 7, 10427 (2016). 